\title{
DISEÑO DE CALCULADORA EN MATLAB PARA EVALUAR MÁQUINAS ELÉCTRICAS ESTÁTICAS EN LABORATORIO VIRTUAL
}

\author{
CALCULATOR DESIGN IN MATLAB TO EVALUATE STATIC ELECTRICAL \\ MACHINES IN VIRTUAL LABORATORY
}

\author{
José L. Pérez Justo"11, Renato Arriola García², Diego Laines Toledo3, \\ Paulo Oliveira Paucar ${ }^{4}$, Margarita Murillo Manrique ${ }^{5}$
}

RECEPCIÓN: 10 DE OCTUBRE DEL 2021

ACEPTACIÓN: 06 DE DICIEMBRE DEL 2021

\section{RESUMEN}

La investigación surgió como iniciativa frente a las dificultades de acceso presencial a los laboratorios debido a la pandemia por el Covid-19. Esta situación obliga a los estudiantes a utilizar las simulaciones como laboratorios virtuales para su aprendizaje; en este caso en concreto para desarrollar los laboratorios del curso de Maquinas Eléctricas de la escuela de ingeniería electrónica de la Universidad Ricardo Palma. En este sentido, la propuesta tiene como objetivo desarrollar una calculadora interactiva enfocada en evaluar los parámetros de transformadores y autotransformadores monofásicos, así como un avance sobre transformadores trifásicos. El procedimiento se sustenta en una programación estructurada en el software de simulación Matlab y en la fundamentación teórica que se desarrolla en la fase de máquinas eléctricas estáticas y en las guías de laboratorio del curso de Máquinas Eléctricas. Los resultados del diseño de la calculadora permitirán obtener parámetros de las pruebas en vacío, pruebas de cortocircuito y los circuitos equivalentes que cumplen con los cálculos eléctricos, características eléctricas y dimensionamiento. Las conclusiones del proyecto determinaron que para un mejor proceso de enseñanza-aprendizaje virtual de máquinas eléctricas estáticas, la calculadora desarrollada en el software Matlab permitirá que el estudiante valide sus resultados teóricos respecto a las variables tensión, corriente y potencia de una máquina, específicamente de los transformadores y autotransformadores monofásicos.

Palabras clave: máquina eléctrica, transformadores monofásicos, autotransformadores, simulación en MATLAB

\begin{abstract}
The research arose as an initiative facing the difficulties of face-to-face access to laboratories due to the COVID-19 pandemic, a situation that forces students to use simulations as virtual laboratories for their learning, in this case to develop the laboratories of the Electrical Machinescourse at the electronic engineering school of the Ricardo Palma University. In this sense, the proposal aims to develop an interactive calculator focused on evaluating the parameters of single-phase transformers and autotransformers, as well as an advance on threephase transformers. The procedure is based on a structured programming in the Matlab simulation software and on the theoretical foundation that is developed in the static electrical machines phase and in the laboratory guides of the Electrical Machines course. The results of the calculator design will provide parameters for the no-load tests, short-circuit tests, and equivalent circuits that meet the electrical calculations, electrical characteristics, and sizing. The conclusions of the project determined that for a better virtual teaching-learning process ofstatic electrical machines, the calculator developed in the Matlab software will allow the student to validate their theoretical results regarding the variables voltage, current and power of a machine. specifically of singlephase transformers and autotransformers.
\end{abstract}

Keywords: electrical machine, single-phase transformers, autotransformers, simulation inMATLAB. 


\section{INTRODUCCIÓN}

Actualmente, el sistema educativo universitario se ha visto afectado por la coyuntura mundial de la pandemia por el Covid-19. Este es un factor que impide a los estudiantes de los diferentes niveles de formación realizar presencialmente experimentos en los laboratorios que los cursos de ingeniería requieren para una formación integral del futuro profesional.

Frente a esta coyuntura, los estudiantes de la asignatura de Máquinas Eléctricas de la escuela profesional de Ingeniería Electrónica decidimos aportar en la realización de una herramienta enfocada al cálculo de transformadores y autotransformadores monofásicos, así como un avance para transformadores trifásicos que a futuro pueda completarse. De esta manera,su uso puede ser un complemento para el desarrollo de laboratorios virtuales en el curso. A pesar deexistir calculadoras virtuales similares de carácter práctico, nuestro diseño tendrá como finalidad principal ser una herramienta pedagógica, para que se constituya en un medio para corroborarresultados hallados teóricamente y compararlos con los resultados esperados en la práctica.

En este sentido, la investigación propone tres objetivos específicos. El primero consisteen organizar los contenidos considerando las competencias que debe lograr el estudiante; el segundo busca esquematizar un formato didáctico de fácil entendimiento y práctico para su manejo y adecuada aplicación, lo que se logrará con el uso del software Matlab y las herramientas queeste posee para crear una interfaz que permita presentar de manera clara los resultados esperados; finalmente, el tercer objetivo, y lo determinamos clave, consiste en desarrollar una interfaz por medio de la herramienta App Designer del programa Matlab.

\section{MATERIALES Y MÉTODOS}

El proceso de diseño considera los transformadores y autotransformadores monofásicos con proyección a incluir transformadores trifásicos. Por esta razón, la organización del proyecto considera como material base las tablas organizacionales que permiten plasmar de manera claray ordenada las fórmulas que se desean incorporar.

En la siguiente sección, se muestran los parámetros utilizados y los que serán calculados, tanto para trasformadores monofásicos como para autotransformadores monofásicos.

\subsection{Parámetros del Transformador Monofásico}

El transformador monofásico es una máquina eléctrica estática. Es uno de los equipos eléctricos más utilizados, que sirve para aumentar o disminuir tensión ycorriente. El esquema se muestra en la figura 1. Debemos tener en cuenta que las pérdidas mecánicas son mínimas y, por lo tanto, puede alcanzar un rendimiento de hastael $98 \%$, y sus únicas pérdidas son el cobre $(\mathrm{Cu})$ y en hierro $(\mathrm{Fe})[1]$.

$2 \quad$ Estudiante de la EPIE-URP r.arriolagar@gmail.com

3 Estudiante de la EPIE-URP. diego.laines@urp.edu.pe

4 Estudiante de la EPIE-URP. paulo.Oliveira@urp.edu.pe

5 Ingeniera Electricista de la Universidad Nacional del Callao, Doctora en Educación, Magíster en Gestión y Docencia Universitaria - UCV, estudios de postgrado en Ingeniería de Sistemas UNI, Bachiller Profesional en Electricidad y Electrónica- ESEP-José Pardo. Doctora Honoris Causa por la UNDAC. Metodóloga en el programaTITES-FI-URP. Docente de las asignaturas de Investigación en Ingeniería, Instalaciones Eléctricas, Máquinas Eléctricas, Taller de Ingeniería Mecatrónica Básica y Circuitos Eléctricos II, en la Escuela de Ing. Civil, Electrónica y Mecatrónica respectivamente. Docente de las asignaturas de Fundamentos de IME e Instalaciones Eléctricas en la Universidad Nacional Tecnológica de Lima Sur. https://orcid.org/0000-0003-2580-0082,ScopusAuthor ID: 57211356705.<mmurillo@urp.edu.pe> 


\subsection{Parámetros del Autotransformador Monofásico}

Algunas aplicaciones requieren modificar niveles de tensión en una porción relativamente pequeña, que tienen en cuenta esta necesidad. Se utiliza un transformador de propósito especial llamado "autotransformador", cuyos esquemas se muestran en laTabla 1. Un autotransformador es una configuración especial de un transformador monofásico que tiene el mismo devanado, tanto en punto de entrada como en el punto de salida [4].

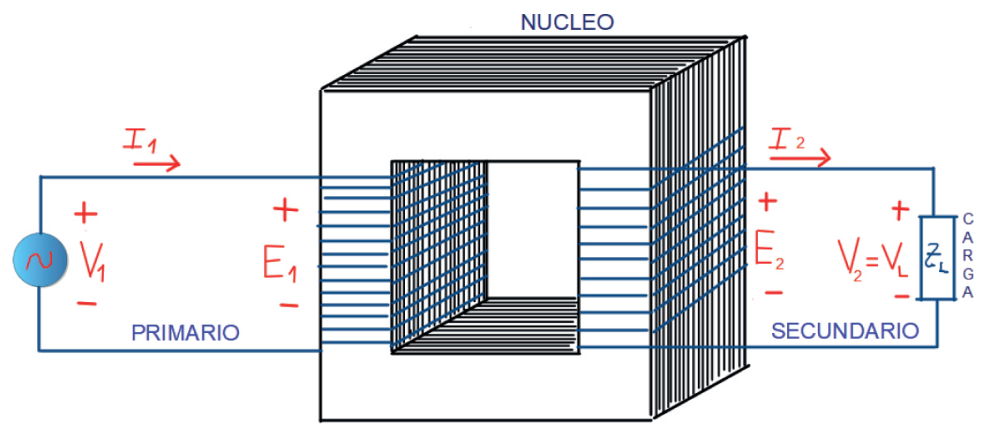

Figura 1. Parámetros del transformador monofásico. Fuente: Recopilado de [3]

Donde:

$V_{1}:$ Tensión de alimentación primaria

$V_{2}$ : Tensión en los bornes de la carga

$g$ : Ganancia en el devanado secundario

$E_{1}:$ Valor eficaz de la fuerza contraelectromotriz

$E_{2}:$ Valor eficaz de la fuerza electromotriz

$I_{1}$ : Corriente nominal aplicado en el primario

$I_{2}$ : Corriente resultante en el secundario

$Z L$ : Impedancia equivalente de la carga

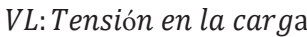

\begin{tabular}{|c|c|}
\hline Autotransformador monofásico reductor & Autotransformador monofásico elevador \\
\hline Figura 2. Esquema eléctrico de un \\
autotransformador reductor
\end{tabular}

Tabla 1. Parámetros eléctricos de un autotransformador monofásico. Fuente: Recopilado de [2]

Donde:

$V_{1}:$ Tensión primaria

$V_{2}:$ Tensión secundaria

$g$ : Ganancia en el devanado secundario 


\section{$N_{1}$ : Espiras en el circuito primario \\ $\mathrm{N}_{2}$ : Espiras en el circuito secundario \\ $N$ t: Espiras totales \\ $m$ : Relación de transformación $\left(m=\frac{V 1}{V 2}\right)$ \\ I: Intensidad de paso \\ $I_{1}$ : Intensidad del circuito primario \\ $I_{2}$ : Intensidad del circuito secundario \\ P: Potencia aparente}

Con los parámetros previamente mencionados procedemos a realizar la calculadora programable en Matlab. Este diseño tendrá la peculiaridad que, una vez ingresados los datos de entrada, esta devolverá los datos de salida esperados. En caso no sea posible, entregará un mensaje que indique que hay datos insuficientes o las correcciones que se deben realizar.

\subsection{Esquema del programa}

El programa se basa en el diagrama de flujo detallado en la figura 2, en el que cada bloquerepresenta una serie de funciones optimizadas de manera que posea una presentación yorganización adecuada para la interfaz que se desea proponer como resultado final.

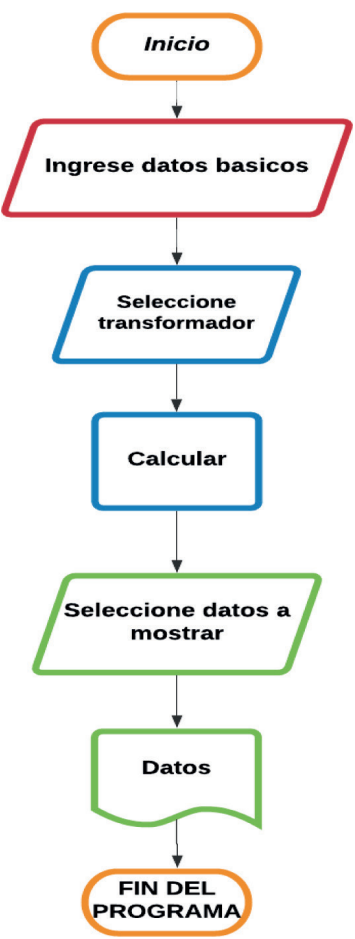

Figura 2. Diagrama de flujo. Elaboración propia

En el primer bloque de la Figura 3 (bloque en rojo), se ingresan los datos del problema que se desee resolver. Estos son los parámetros generales como voltajes, potencia, entre otros,y son obtenidos de manera didáctica por la interfaz del programa. Cabe mencionar que no es necesario ingresar todos los datos de la tabla didáctica "selección dato de entrada". 
Las unidades están definidas de acuerdo con el parámetro seleccionado y se necesita la confirmación del botón grabar para guardar el dato. Una vez que se tienen los datos de ingreso, se selecciona el tipo de transformador con el que se desea trabajar. Esto permiteelegir los bloques de ecuaciones programados idealmente para el caso a evaluar y esperar la confirmación del botón calcular para proceder con los mismos. Estos procedimientos hacen referencia a la segunda etapa de la Figura 3 (bloque azul).

La etapa final está demarcada en la Figura 3 (bloque verde), que nos muestra la presentación de los parámetros que se desean conocer.

La ventana didáctica de la interfaz permite seleccionar el dato de salida y mostrar los valores obtenidos. En la versión final del programa, se incluirá también una imagen con la fórmula utilizada para obtener dicho resultado.

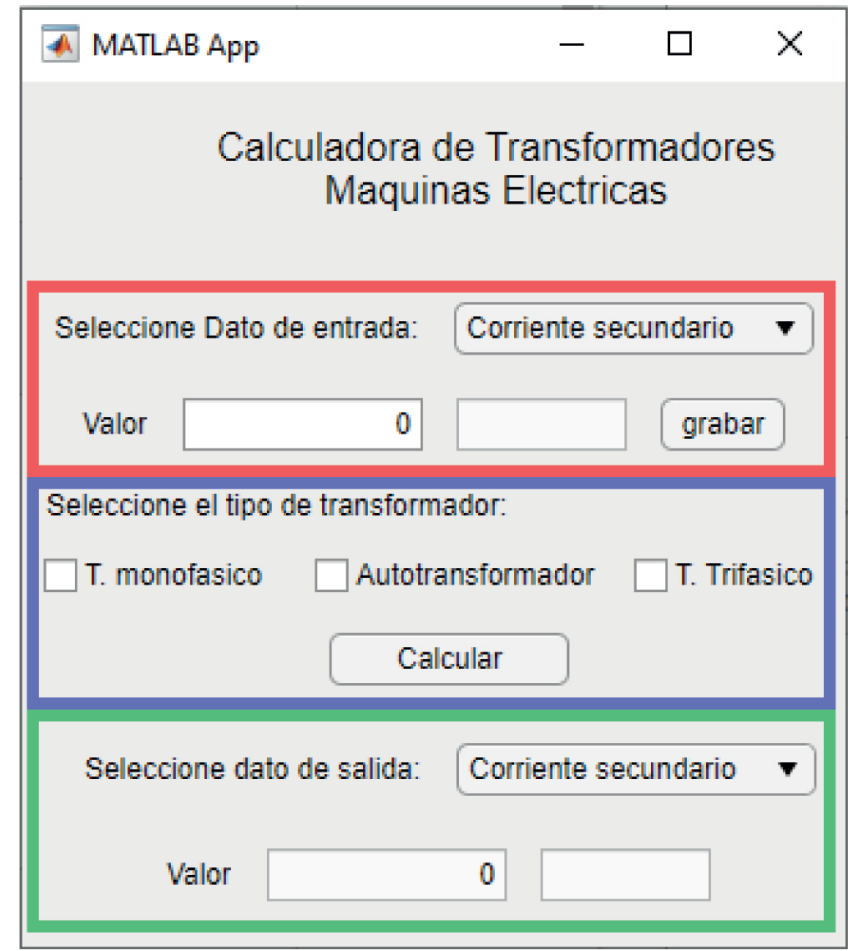

Figura 3. Interfaz en App Designer. Elaboración propia

\section{RESULTADOS}

\subsection{Resultados del transformador monofásico}

Datos teóricos de entrada de un transformador monofásico:

$V_{1}=220 \mathrm{~V}$

$V_{2}=24 \mathrm{~V}+10 \%=26.4 \mathrm{~V}$

$F=60 \mathrm{~Hz}$

$P=500 \mathrm{VA}$ 
Ahora realizaremos los cálculos teóricos en la Tabla 2 del resto de los parámetros eléctricos del transformador monofásico en función de la tabla 5 (anexo).

\begin{tabular}{|c|c|}
\hline Tensión primaria & $230 \mathrm{~V}$ \\
\hline Intensidad primaria & $2.1 \mathrm{~A}$ \\
\hline $\begin{array}{c}\text { Número de espiras enel } \\
\text { primario }\end{array}$ & 350 espiras \\
\hline $\begin{array}{c}\text { Diámetro del hilo } \\
\text { primario }\end{array}$ & $0.85 \mathrm{~mm}$ \\
\hline Tensión secundaria & $26.4 \mathrm{~V}$ \\
\hline $\begin{array}{c}\text { Intensidad secundaria } \\
\text { Número de espiras enel } \\
\text { secundario }\end{array}$ & 40 espiras \\
\hline $\begin{array}{c}\text { Diámetro del hilo } \\
\text { secundario }\end{array}$ & $2.24 \mathrm{~mm}$ \\
\hline Potencia & $500 \mathrm{VA}$ \\
\hline Espiras por voltio & $1.52 \mathrm{E} / \mathrm{V}$ \\
\hline Frecuencia & $60 \mathrm{~Hz}$ \\
\hline Sección del núcleo & $25.3 \mathrm{~cm}$ \\
\hline
\end{tabular}

Tabla 2. Parámetros hallados del transformador monofásico Fuente: Recopilado de [2]

Los datos obtenidos en la calculadora de transformadores, mediante los datos de entradateórica del transformador monofásico, se muestran en la figura 4.

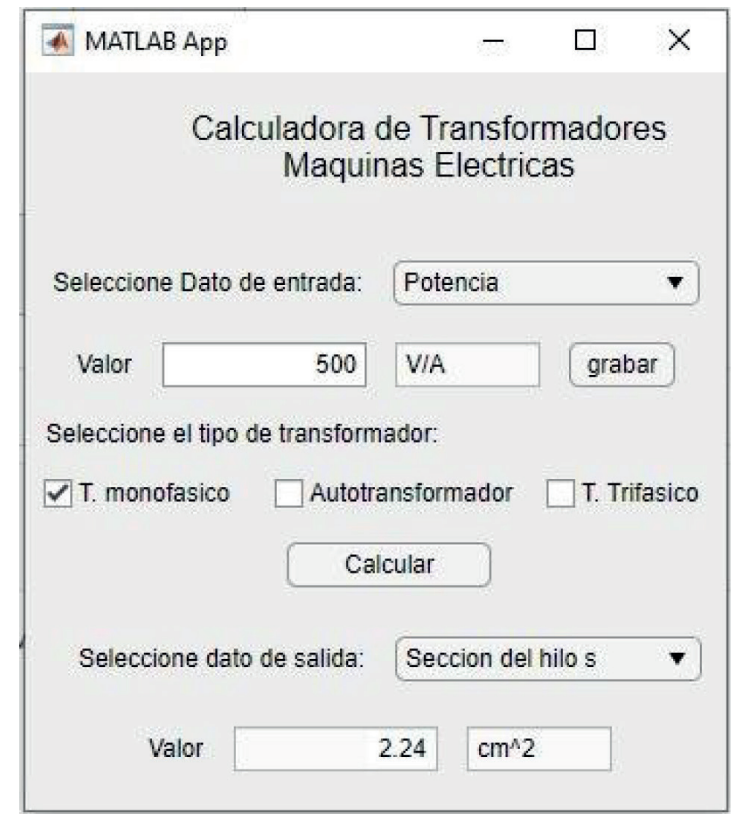

Figura 4. Cálculos obtenidos de un transformador monofásico. Elaboración propia 


\subsection{Resultados del autotransformador monofásico}

Datos teóricos de un autotransformador monofásico reductor:

$V_{1}=220 \mathrm{~V}$

$V_{2}=127 \mathrm{~V}+5 \%=133 \mathrm{~V}$

$F=60 \mathrm{~Hz}$

$P=2000 V A$

Ahora realizaremos los cálculos teóricos en la Tabla 3 del resto de los parámetros eléctricos del autotransformador monofásico reductor en función de la tabla 6 (anexo):

\begin{tabular}{|c|c|}
\hline & $\begin{array}{l}\text { Autotransformador monofásico } \\
\text { reductor }\end{array}$ \\
\hline Tensión primaria & $220 \mathrm{~V}$ \\
\hline Tensión secundaria & $127 \mathrm{~V}$ \\
\hline Potencia & $2000 \mathrm{VA}$ \\
\hline Frecuencia & $60 \mathrm{~Hz}$ \\
\hline Sección del núcleo & $31.82 \mathrm{~cm}^{2}$ \\
\hline Espiras por voltio & 1.21 espiras por cada voltio \\
\hline $\begin{array}{l}\text { Relación de } \\
\text { transformación }\end{array}$ & 1.73 \\
\hline Intensidad de paso & $6.3 \mathrm{~A}$ \\
\hline $\begin{array}{c}\text { Intensidad del circuito } \\
\text { primario }\end{array}$ & $8.7 \mathrm{~A}$ \\
\hline $\begin{array}{c}\text { Intensidad del circuito del } \\
\text { secundario }\end{array}$ & $15 \mathrm{~A}$ \\
\hline $\begin{array}{c}\text { Espiras del circuito } \\
\text { primario }\end{array}$ & 105 espiras \\
\hline $\begin{array}{c}\text { Espiras del circuito } \\
\text { secundario }\end{array}$ & 161 espiras \\
\hline $\begin{array}{c}\text { Diámetro promedio } \\
\emptyset_{1-2}=\begin{array}{c}\emptyset_{1}+\emptyset_{2} \\
2\end{array}\end{array}$ & $1.38 \mathrm{~mm}$ \\
\hline
\end{tabular}

Tabla 3. Parámetros hallados del autotransformador monofásico reductor. Fuente: Recopilado de [2]

Los datos obtenidos en la calculadora de transformadores, mediante los datos de entradateórica del autotransformador monofásico reductor, se muestran en la figura 5 . 


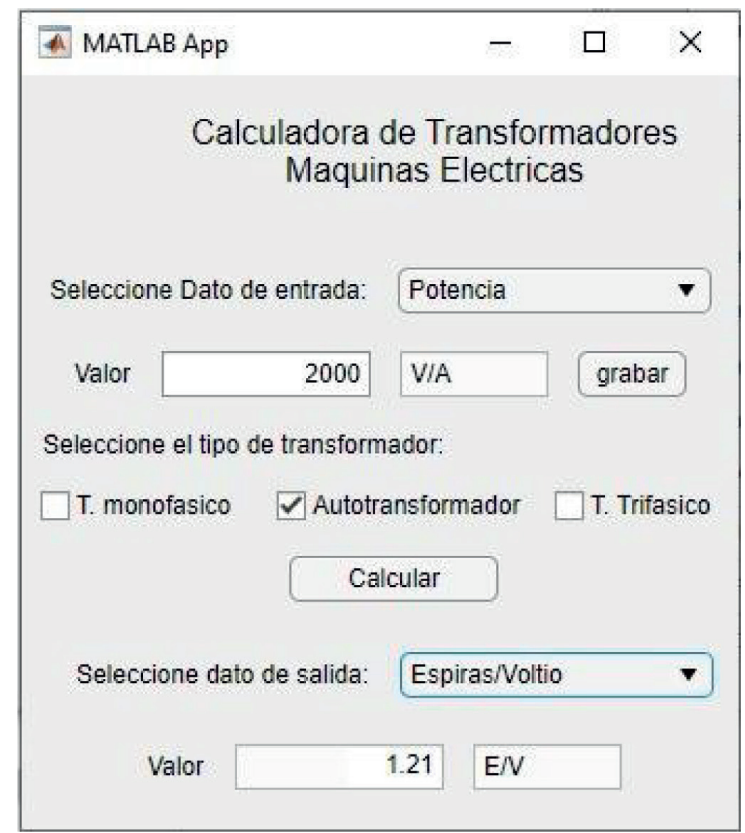

Figura 5. Cálculos obtenidos de un autotransformador monofásico reductor. Elaboración propia

Datos teóricos de un autotransformador monofásico elevador:

$\mathrm{V}_{1}=220 \mathrm{~V}$

$\mathrm{V}_{2}=380 \mathrm{~V}+5 \%=399 \mathrm{~V}$

$\mathrm{F}=60 \mathrm{~Hz}$

$\mathrm{P}=1500 \mathrm{VA}$

Ahora realizaremos los cálculos teóricos Tabla 4 del resto de los parámetros eléctricosdel autotransformador monofásico elevador en función de la Tabla 6 (anexo):

\begin{tabular}{|c|c|}
\hline & $\begin{array}{c}\text { Autotransformador } \\
\text { monofásico elevador }\end{array}$ \\
\hline Tensión primaria & $220 \mathrm{~V}$ \\
\hline Tensión secundaria & $399 \mathrm{~V}$ \\
\hline Potencia & $1500 \mathrm{VA}$ \\
\hline Frecuencia & $60 \mathrm{~Hz}$ \\
\hline Sección del núcleo & $27.59 \mathrm{~cm}^{2}$ \\
\hline Electrón por voltio & 1.39 Espiras por cadavoltio \\
\hline $\begin{array}{c}\text { Relación de } \\
\text { transformación }\end{array}$ & 0.58 \\
\hline Intensidad de paso & $3 \mathrm{~A}$ \\
\hline $\begin{array}{c}\text { Intensidad del circuito } \\
\text { primario }\end{array}$ & $6.8 \mathrm{~A}$ \\
\hline
\end{tabular}




\begin{tabular}{|c|c|}
\hline $\begin{array}{c}\text { Intensidad del circuitodel } \\
\text { secundario }\end{array}$ & $3.8 \mathrm{~A}$ \\
\hline $\begin{array}{c}\text { Espiras del circuito } \\
\text { primario }\end{array}$ & 307 espiras \\
\hline $\begin{array}{c}\text { Espiras del circuito } \\
\text { secundario }\end{array}$ & 250 espiras \\
\hline Diámetro promedio & $0.93 \mathrm{~mm}$ \\
$\emptyset_{\mathbf{1}-\mathbf{2}}=\emptyset_{\mathbf{2}}$ & \\
\hline
\end{tabular}

Tabla 4. Parámetros hallados de un autotransformador monofásico elevador. Fuente: Recopilado de [2]

Los datos obtenidos en la calculadora de transformadores, mediante los datos de entradateórica del autotransformador monofásico elevador, se muestran en la figura 5.

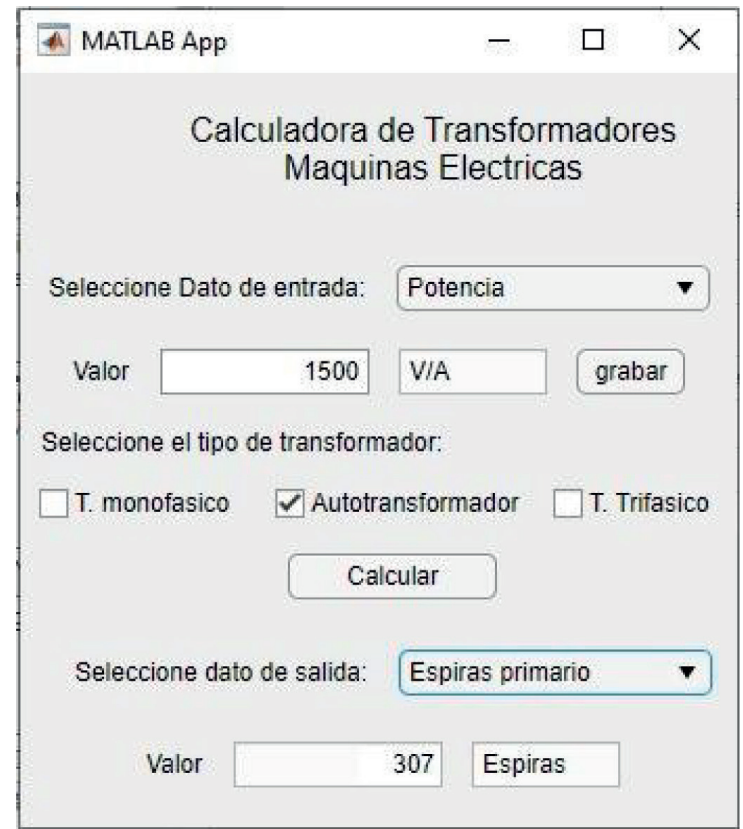

Figura 5. Cálculos obtenidos de un autotransformador monofásico elevador. Elaboración propia

\section{DISCUSIÓN}

Los softwares usados en diseńos de simuladores eléctricos profesionales tienen un costo elevado de licencia, lo que los hace de acceso limitado para la gran mayoría de estudiantes. Por este motivo, se utilizan los softwares libres. Al hacer un análisis respecto a estos se pudo comprobar que estos últimos no poseen el mismo poder computacional o las interfaces que permitan aplicaciones amplias. Sin embargo, varios estudios validan que para aplicaciones pedagógicas es factible su uso. 
En la programación existe estándares y normativas que se deben cumplir en función de la aplicación y extensión que se desee dar al código programado que se presenta. Teniendo encuenta lo mencionado, un código debe ser modulado y estructurado para que permitarealizar mejoras para futuros usos en base al código fuente.

\section{CONCLUSIONES}

El desarrollo de la investigación nos permite concluir que, además de la aplicación didácticadel respectivo programa basado en cálculos con valores reales, se podrán visualizar todos los modelos de transformadores de distribución monofásicos (trifásico a futuro), considerando sus respectivos parámetros eléctricos y constructivos. De este modo, se facilita en gran medida el proceso de enseñanza-aprendizaje en materias como sistemas de alta tensión, diseño de instalaciones industriales y especialmente máquinas eléctricas.

Se considera necesario optimizar un sistema de filtrado de datos que permita validar si los valores ingresados son compatibles con las especificaciones de los cálculos teóricos programados en el software Matlab. Teniendo en cuenta que la calculadora de transformadores será usada por estudiantes de todos los ciclos y niveles, se puede irperfeccionando en la medida en que se puedan validar las guías del laboratorio.

\section{REFERENCIAS}

[1] J. F. Mora, Máquinas Eléctricas, 4ta ed. Madrid: McGraw-Hill, 2008.

[2] M. Álvarez Pulido, Transformadores, 1st ed. México: Alfaomega Grupo Editor, 2009.

[3] M. Capcha Aira, Máquinas Eléctricas, 5th ed. Lima: Editorial Ciencias, 2009.

[4] P. Ponce Cruz, Máquinas Eléctricas, 2nd ed. México: Alfaomega Grupo Editor, 2016. 


\section{ANEXOS}

Tabla 5. Fórmulas para hallar los parámetros de un transformador monofásico.

\begin{tabular}{|c|c|c|c|c|c|}
\hline \multirow{4}{*}{ 产 } & Espiras/Voltio & $\begin{array}{l}\text { Numero de espiras } \\
\text { en el devanado } \\
\text { primario }\end{array}$ & $\begin{array}{l}\text { Números de espiras } \\
\text { en el devanado } \\
\text { secundario }\end{array}$ & $\begin{array}{c}\text { Sección del } \\
\text { núcleo }\end{array}$ & $\begin{array}{c}\text { Intensidad del } \\
\text { devanado } \\
\text { primario }\end{array}$ \\
\hline & $E / V=\frac{34}{\sqrt{P}}$ & $N_{1}=(E / V) * V_{1}$ & $N_{1}=(E / V) * V_{2}$ & $\begin{array}{c}S=\frac{V_{1} * 100}{2.6 * N_{1}} \\
\circ \\
S=\frac{V_{2} * 100}{2.6 * N_{2}}\end{array}$ & $I_{1}=\frac{P}{V_{1}}$ \\
\hline & $\begin{array}{l}\text { Intensidad del } \\
\text { devanado } \\
\text { secundario }\end{array}$ & $\begin{array}{l}\text { Sección del hilo } \\
\text { del devanado } \\
\text { primario }\end{array}$ & $\begin{array}{c}\text { Sección del hilo del } \\
\text { devanado } \\
\text { secundario }\end{array}$ & $\begin{array}{c}\text { Diámetro del hilo } \\
\text { del devanado } \\
\text { primario }\end{array}$ & $\begin{array}{l}\text { Diámetro del hilo } \\
\text { del devanado } \\
\text { secundario }\end{array}$ \\
\hline & $I_{2}=\frac{P}{V_{2}}$ & $S_{1}=\frac{l_{1}}{J}$ & $\begin{array}{c}\quad S_{2}=\frac{\tau_{2}}{J} \\
\text { J: Densidad de } \\
\text { corriente }\end{array}$ & $\emptyset_{1}=\sqrt{\frac{S_{1}}{0.785}}$ & $\emptyset_{2}=\sqrt{\frac{S_{2}}{0.785}}$ \\
\hline
\end{tabular}

Fuente: Recopilado de [2]

Tabla 6. Fórmulas para hallar los parámetros de un autotransformador monofásico elevador y reductor

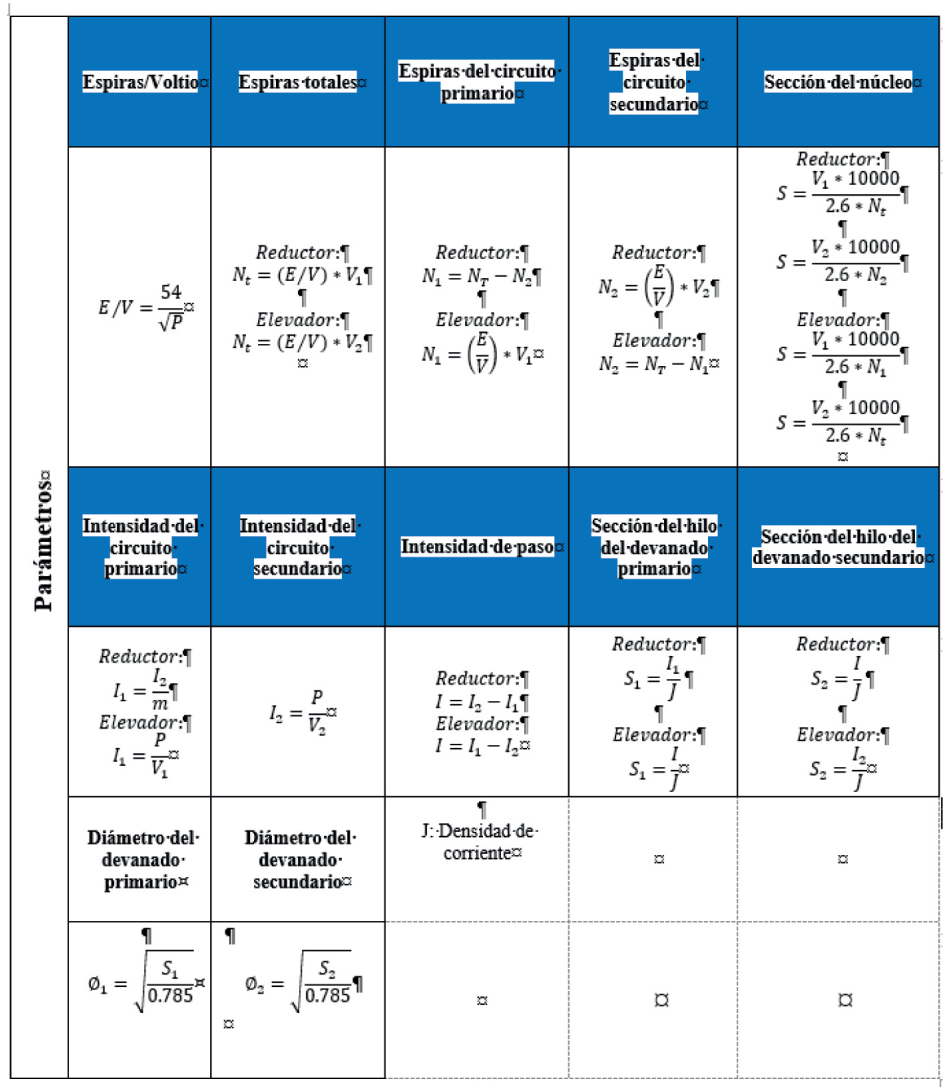

Fuente: Recopilado de [2] 